\title{
Falls among the elderly: distinguishing indoor and outdoor risk factors in Canada
}

\author{
Jennifer L O'Loughlin, Jean-François Boivin, Yvonne Robitaille, Samy Suissa
}

Recognition of the complex aetiology of falls has led some researchers to limit their studies on the identification of risk factors to subcategories of falls such as injurious falls, falls requiring treatment, recurrent falls, falls in the home, or falls related to stairs. Other investigators have collected data on all falls and then classified them according to cause, ${ }^{1}$ severity of injury sustained, ${ }^{2}$ or activity engaged in at the time of the fall. ${ }^{3}$ However, the usefulness of these approaches for the purposes of research and prevention remains to be seen.

We report here a simple fall classification by location (indoors or outdoors) and a comparison of the risk factors for falls in each location. The suggestion that risk factors for indoor and outdoor falls differ is intuitively appealing because of the different environmental challenges in these settings, the distinct physical attributes and coping strategies required to meet these challenges and the very different activities engaged in indoors and outdoors. There is indeed some recognition that the causes of indoor and outdoor falls may differ. ${ }^{1}$

Public Health Unit, Montreal General

Hospital, 1616 boul René-Levesque 0 .,

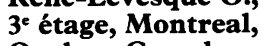
Quebec, Canada

H3H IP8

J L O'Loughlin

Y Robitaille

Department of

Epidemiology and

Biostatistics,

McGill University,

Montreal, Canada

J-F Boivin

S Suissa

Correspondence to: Dr J O'Loughlin.

Accepted for publication May 1994
Methods: In 1987-88 we conducted a study among non-institutionalized people aged 65 years or more living in Montreal, Canada. Subjects were randomly selected from the 1985 Quebec electoral list. After a baseline questionnaire had been administered in their homes, subjects were interviewed by telephone once every four weeks for 48 weeks. In the baseline interview, data were collected on those exposures that were not expected to fluctuate substantially over time, including demographic variables, chronic health problems, height and weight, lifestyle, and balance and mobility. A history of falls in the previous year was also obtained.

Data on time-varying exposures, including the level of physical activity, alcohol and medication use, dizziness, and bed- and activitylimitation days, were collected in the baseline and follow up interviews. Also during each follow up interview, data on falls sustained since the previous interview were collected. Classification of falls as indoor or outdoor was based on subject self reports of the location of the fall, and confirmed through comparison with subjects' verbatim descriptions of their falls. Detailed study methods including selection criteria, definitions of study variables, and analytic methods were reported earlier. ${ }^{4}$

Because some subjects go out very little because of their health or for other reasons, risk factors for falls which are also markers of inability to go outdoors would be associated with the risk of indoor, but not outdoor falls. The difference between risk factors for indoor and outdoor falls would then be due, not to those risk factors, but to the lack of opportunity to fall outdoors. To take this into account, we compared risk factors for indoor and outdoor falls in a dataset restricted to those four week intervals of observation during which subjects had engaged in more than 10 physical activities in the week preceding the interview. We believed that this variable represented the best indicator of "outdoor exposure" because of the strong and statistically significant correlations with other variables in the dataset also judged to be plausible markers of outdoor exposure (for example, difficulty walking $400 \mathrm{~m}$ without resting, use of homecare services, number of chronic health problems, physical activity compared to peers, and usual level of physical effort in daily activities). We used pooled logistic regression ${ }^{5}$ for our multivariate analyses.

Results: Among 556 persons aged 65 years or more selected from the electoral list, 417 com-

Risk factors for indoor and outdoor falls among elderly people living in the community in west-central Montreal, 1987-88

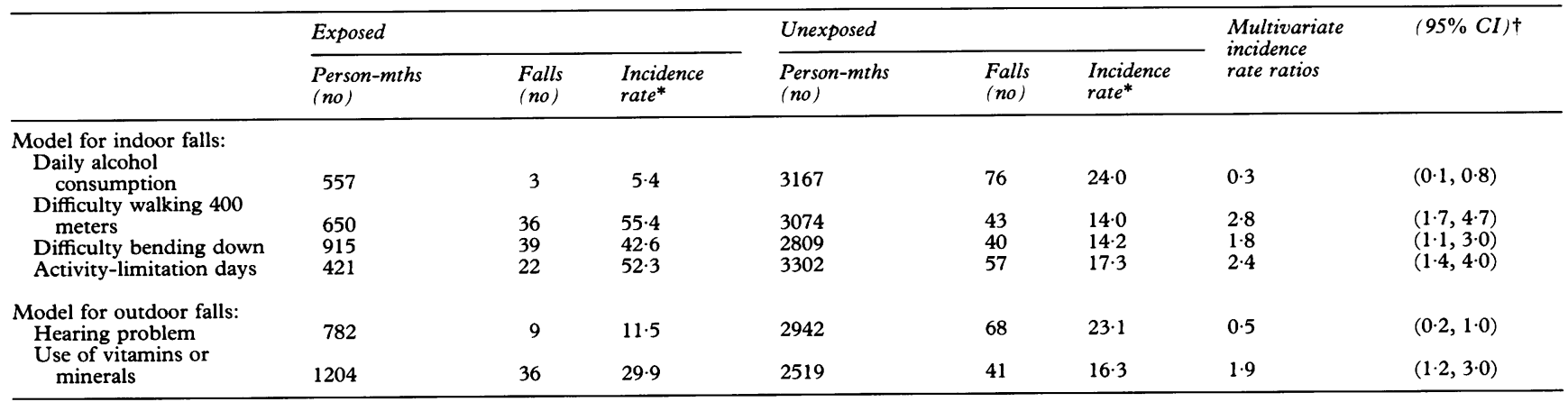

* Number of falls per 1000 person-months.

+ All factors met the significance criterion of $p \leqslant 0 \cdot 05$ 
pleted the baseline interview, and $90 \%$ or more of these completed each of the follow up interviews. In the 3724 person-months during which subjects had "outdoor exposure", 155 falls were reported. The incidence rates of indoor and outdoor falls were 21.2 and 20.4 falls per 1000 person-months, respectively. The table shows that the risk factors differed for indoor and outdoor falls. Our results, if confirmed, suggest that indoor and outdoor falls should be studied as separate outcomes. This distinction might eventually prove useful for the design of preventive interventions.
This research was supported by the Fonds de la Recherche en Santé du Québec (grant 158085).

1 Hale WA, Delaney MJ, McGaghie WC. Characteristics and predictors of falls in elderly patients. $\mathcal{f}$ Fam Prac 1992;34:577-81.

2 Tinetti ME. Factors associated with serious injury during falls by ambulatory nursing home residents. $\mathcal{F}$ Am Geriatr Soc 1987;35:644-8.

3 Tinetti ME, Speechley M, Ginter SF. Risk factors for falls among elderly persons living in the community. $N$ Engl $f$ Med 1988:319:1701-7.

4 O'Loughlin J, Robitaille Y, Boivin JF, Suissa S. The incidence of and risk factors for falls and injurious falls among community-dwelling elderly. Am $\mathcal{f}$ Epidemiol 1993;137:342-54.

5 D'Agostino RB, Lee M, Belanger AJ, Cupples LA, Anderson $\mathrm{K}$, Kannel WB. Relation of pooled logistic regression to time dependent Cox regression analysis: The Framingham Heart Study. Stat Med 1990;9:1501-15.

\title{
Remembering fractures: fracture registration and proband recall in southern Sweden
}

\author{
Brynjólfur Jónsson, Per Gärdsell, Olof Johnell, Inga Redlund-Johnell, Ingemar Sernbo
}

This study aimed to examine fracture recall and to compare different sources of information on fracture registration both within the same population and between areas. The study was carried out in southern Sweden. Altogether 782 individuals of both sexes from the city of Malmö, and 486 residents from the nearby rural community of Sjöbo were invited to participate in a population based study. A total of $570(73 \%)$ from Malmö and 391 (80\%) from Sjöbo participated. For all subjects, fracture data were obtained from three different sources - one, the probands' own recollection of previous fracture episodes and the other two, patient records. In addition, the age at the time of fracture and the severity of the trauma that caused the fracture were recorded. Where fractures were sustained and treated elsewhere and were not on our records, records from other hospitals were not studied.

Results: In Malmö, 155 of the subjects had sustained a total of 252 fractures. In Sjöbo, 75 probands had sustained 91 fractures. In Malmö $40 \%$ and in Sjöbo $22 \%$ of the fractures had been forgotten by the probands and were discovered from patient records (figure). In both communities, half of those with fractures not detailed in the records had either been treated elsewhere or had moved to their present residence since their fractures. Almost $40 \%$ of all fractures had been forgotten by the probands: in men $60 \%$ of fractures of the hand and foot and in women $22 \%$ of fractures of the distal end of the radius had been forgotten.

The median time that had elapsed between forgotten fractures and the interview was longer than that for remembered fractures: in Malmö these times were 15 and 9 years, respectively $(p=0.001$, Mann-Whitney) and in Sjöbo, 14 and 9 years, respectively $(p=0.001)$. Fractures caused by more severe trauma were signific- antly more likely to have been forgotten in Malmö - these high energy fractures were more common in men.

Discussion: The data indicate that there will be considerable under-reporting if only one source of information is used in the screening of fractures. The cause of the discrepancy between registration and recall in the two communities may be less accurate registration in Sjöbo because people living in the outskirts of the community might have sought medical care outside of the health district.

In prospective studies, medical records have been found to confirm fracture history in all cases in women, ${ }^{1}$ but over reporting of self reported fractures has been found in $20 \%$ of cases among elderly women. ${ }^{2}$ Even in cross sectional studies similar to ours there was a tendency to over report fractures. ${ }^{3}$ Because the accuracy of a positive fracture history outside the study health districts was not examined, some of the fractures reported but not recorded may be false positives. An alternative explanation might be either better recall in rural probands, who also had fewer fractures, and fewer fractures per fractured individual, ${ }^{4}$ together with possibly more tangible effects of the fractures on the working capacity in a farming population.

Women in this study had forgotten more than one fifth of the fractures of the distal end of the radius. This is in line with the study of Åkeson et al, 5 in which $14 \%$ of 36 women did not remember having sustained a wrist fracture 0-35 (mean 10) years earlier, and additionally $17 \%$ had forgotten which side they had broken.

The time elapsed since the fracture seems to be of importance for recall. In this study the median time elapsed after a remembered fracture was 9 years, whereas it was 14-15 years for 